\title{
PROKLA-Redaktion
}

\section{Editorial: Biokapitalismus}

Die Diskussion über gesellschaftliche Naturverhältnisse hat vielfach das gesellschaftliche Verhältnis zur äußeren Natur thematisiert. Dabei geht es um die herrschaftliche Aneignung der Natur. Die Befürchtung, die bereits von Marx geäußert wurde, dass nämlich der Kapitalismus die natürlichen Lebensgrundlagen der Menschen und ihrer Gesellschaft untergräbt und zerstört, hat nichts von ihrer Bedeutung und Aktualität verloren. Wenn neuerdings nach einem Ausdruck von Paul Crutzen vom Anthropozän die Rede ist, dann ist damit das erdgeschichtliche Moment gemeint, in dem die Menschen eine alles Leben beeinflussende geologische Kraft geworden sind. Doch ist das keine Feststellung eines bloß positiven Sachverhalts. Vielmehr hat sich die schon von Marx beobachtete Tendenz, dass es keine von Menschen unberührte Natur mehr gibt, radikal fortgesetzt. Sie haben unter kapitalistischen Bedingungen in derart beschleunigter Weise die Erdkruste aufgewühlt und in die natürlichen Kreisläufe eingegriffen, dass es in vielerlei Hinsicht zu dramatischen Rückwirkungen auf das gesellschaftliche Leben kommt: Erwärmung von Atmosphäre und Meeren, Klimaturbulenzen, Anhebung der Meeresspiegel, Überfischung oder Desertifikation, Verlust der Biodiversität, Degradation der Böden oder Zersiedelung, Vergiftung und Chaotisierung natürlicher Umwelten durch Extraktion der Rohstoffe oder Vergiftung durch Abfälle sind jeweils Folgen einer weitgehend ungezügelten Ausplünderung der Natur - vielfach zum Nutzen der Bereicherung von Wenigen. In einer Zeit, in der es ein globales Wissen um jeden Thunfischschwarm in den Weltmeeren gibt, in der auch der letzte Baum der weltweiten Regenwälder kartografisch erfasst und gezählt ist, erweist sich die Menschheit dennoch nicht in der Lage, die gesellschaftlichen Naturverhältnisse zu reorganisieren und zu demokratisieren. Der Begriff der Nachhaltigkeit, ein Begriff der frühen Aufklärung, ist heute den Begriffen der Freiheit, Gleichheit und Solidarität durchaus gleichrangig. Er konnte in den 1980er und 1990er Jahren mit dem Brundtland-Bericht und der Weltumweltkonferenz in Rio wichtige Impulse für die öffentliche Diskussion und die politische Praxis geben. Doch wurde er alsbald ein Allerweltsbegriff der internationalen Organisationen und nationalen Regierungen, dessen Bedeutung nahezu ins Gegenteil verkehrt wurde: Nachhaltiges Wachstum oder nachhaltige Haushaltssanierung stehen für eine neoliberale Politik, die sich um die Folgen für die Natur nicht bekümmert. Wie ambivalent, wie zynisch sich die Dinge entwickeln, zeigt sich daran, dass die amerikanische Ökonomie dafür gelobt wird, die Krise durch billige Energie überwunden zu haben, also durch Schiefergas und Teeröl. Der US-Präsident kann gleichzeitig auf dem G20-Treffen von Australiens Premierminister verlangen, 
dass sein Land die $\mathrm{CO}_{2}$-Emissionen reduziert. Als zynisch können auch all jene Versuche bezeichnet werden, die die Lösung von Umweltproblemen durch Techniken des Geo-Engineering oder der Gentechnik erwarten. Seit Jahrzehnten gibt es die Versuche der großen Agrar- und Lifescience-Unternehmen, ein Eigentumsmonopol über die genetische Vielfalt und die natürliche Reproduktionsfunktion von Pflanzen und Tieren zu erlangen. Unternehmen aus dem globalen Norden sammeln Pflanzen aller Art, um die daraus gewonnene DNA zu patentieren und ein Monopol auf entsprechende Nutz- und Heilpflanzen zu errichten. Die Bauern und die indigenen Bevölkerungen werden ihres Wissens ebenso beraubt wie ihrer Ressourcen enteignet, da ihnen aufgrund eigentumsrechtlicher Regelungen die weitere Nutzung solcher Pflanzen verwehrt wird. Bauern werden auf diese Weise und mit dem Versprechen auf höhere Erträge gedrängt, die Getreide- oder Reissaat bei Gentech-Unternehmen zu kaufen. Dies verbindet sich mit Kreisläufen von Kreditfinanzierung und Verschuldung.

Was mit Blick auf die äußere Natur festgestellt werden kann, gilt auch mit Blick auf die innere Natur, den menschlichen Körper. Das Verhältnis zum menschlichen Leben hat sich durch Medizin und Humangenetik verändert. Das Innere des menschlichen Körpers war, wie Petra Gehring feststellt, über Jahrtausende „unvernutzbares Niemandsland“. Die Hautgrenze wurde schon im 18. Jahrhundert überschritten. Mit dem Zugriff auf Körperstoffe wie Blut, Eizellen, Sperma, Organe, Gewebe verändert sich das gesellschaftliche Naturverhältnis zum Körper offensichtlich tief greifend. Von dem individuellen Körper wird abstrahiert, er wird auf biologische Funktionen reduziert und kommerzialisiert (vgl. Gehring 2006). Seit der Entdeckung der rekombinanten
DNA-Technologie 1973 findet ein zunehmender gentechnisch-medizinischer $\mathrm{Zu}$ griff auf das Leben als solches - einschließlich des menschlichen Lebens - statt und führt zur Entwicklung eines umfassenden industriell-medizinischen Komplexes, der Biotech-Unternehmen (oftmals Ausgründungen aus Universitäten, die sich auf Risikokapital-GeberInnen stützen können), die pharmazeutische Industrie, öffentliche Forschungseinrichtungen und Hospitäler umfasst. Durch die Verbindung von Genetik und Informationstechnologie ist es gelungen, das menschliche Leben in der abstrakten Form von Informationen zu analysieren und zu kartografieren, das Leben (in der Form von DNA-Sequenzen, Zellen, Gewebe, Embryonen, Organen) als Eigentum zu verrechtlichen und der Verwertung zu unterwerfen. Dies gibt global operierenden Unternehmen Anlass dazu, großflächig nicht nur die DNA von Pflanzen zu erfassen, sondern auch diejenige von großen Bevölkerungsgruppen. Weil die DNA aber allein noch wenig bedeutet, wenn die Erkenntnisse auch von der pharmazeutischen Industrie für die Entwicklung von Arzneimitteln genutzt werden sollen, wird versucht, auch die Krankheitsvorfälle und die familiären Zusammenhänge der Erkrankten mit zu erfassen. Daraus entstehen vielfache Eigentumskonflikte zwischen denen, von denen die Daten erhoben werden, den „Quellen“, denjenigen, die Banken von Daten und Gewebeproben anlegen und schließlich den Unternehmen, die darauf gestützt Medikamente entwickeln (vgl. Sunder Rajan 2009). Es kommt zu großflächigen Projekten des „life mining“ und des genetischen „bio-farming“. Aber der Zugriff der kapitalistischen Ökonomie ist nicht auf die Gene begrenzt.

Neben und teilweise verbunden mit der Humangenetik haben sich weitere Technologien in der Medizin entwickelt. Diese 
reproduktionsmedizinischen Techniken verändern ebenfalls die Anordnung von Gewohnheiten und Selbstverständlichkeiten und erzeugen neue Bedürfnisse und Konsummuster. Noch bis vor wenigen Jahrzehnten galt als natürliche Gegebenheit, dass eine Frau, die ein Kind gebärt, auch die leibliche Mutter dieses Kindes ist. Sofern der Mann nicht zeugungs-, die Frau nicht gebärfähig war, blieb das Paar zunächst kinderlos - es sei denn, es adoptierte Kinder. Künstliche Befruchtung hat die Situation radikal verändert. Ein großer medizinisch-ökonomischer Apparat ist darum herum entstanden: Kinderwunschkliniken, Nahrungsmittel, hormonelle und chirurgische Eingriffe, sogenannte Eizellvorsorge (also Lagerung eingefrorener Eizellen), Samenbanken, umfassende rechtliche Regelungen, die auch das Verhältnis von biologischer und sozialer Elternschaft regulieren. Mit der Invitrofertilisation und neuen, pränatalen diagnostischen Möglichkeiten, die die Gentechnik zur Verfügung stellt, ist die Sorge verbunden, dass es zu eugenischen Maßnahmen kommen kann, weil Eltern vermeiden wollen, dass ein Kind behindert ist. Besonders umstritten ist aus einer kritischen disability-Pespektive dabei die gesellschaftliche Wirkung der pränatalen Diagnostik (PID), die in Deutschland seit 2010 im Embryonenschutzgesetz verankert ist. Bereits heute werden in Deutschland ungefähr 90 Prozent der Föten, bei denen Trisomie 21 diagnostiziert wird, abgetrieben. Für Frauen, denen nach der Fruchtwasseruntersuchung ein behindertes Kind diagnostiziert wird, kann das eine Situation bedeuten, in der sie sich für oder gegen eine Spätabtreibung entscheiden müssen. Dabei handelt es sich um eine künstlich eingeleitete Geburt, die jedoch weder medizinisch noch juristisch genau bestimmt ist. Dem Fötus wird entweder Kaliumchlorid injiziert oder die Blutversorgung über die Nabelschnur wird unterbunden. Es kommt allerdings auch zu Fällen, in denen der Fötus lebend zur Welt kommt und erst nach der Geburt stirbt. In sogenannten Frauenzeitschriften wie der Brigitte, aber auch in verschiedensten Feuilletons werden Erfahrungsberichte diskutiert. Deutlich wird dabei, dass Frauen (und ggf. auch ihrE PartnerIn), die sich für eine Spätabtreibung entscheiden, wie auch Frauen, die sich bewusst dagegen entscheiden, einem hohen gesellschaftlichen (ärztlichen, familiären, freundschaftlichen, beruflichen) Druck ausgesetzt sehen, der anscheinend einem „doublebind“-Charakter entspricht: Entscheiden sie sich für das Kind, stoßen sie in Teilen ihrer Umgebung auf Ablehnung; entscheiden sie sich dagegen, blüht ihnen das Gleiche. Hinzu kommt der oft enorm belastende zeitliche Zwang, unter dem eine solche Entscheidung getroffen werden muss. Feministische Einschätzungen zur PID fallen - nicht besonders überraschend - höchst unterschiedlich aus.

Die angeschnittenen Diskussionen finden auch statt vor dem Hintergrund biopolitischer Befürchtungen: Über kurz oder lang, so erwarten es manche, kommt es aus versicherungstechnischen Gründen zu einem Gen-Screening: Wer trotz aller Möglichkeiten des Wissens ein in irgendeiner Hinsicht sozial als defizient bewertetes Kind (Behinderung, IQ, Geschlecht) zur Welt bringt, ist selbstverantwortlich und wird von den Krankenkassen nicht unterstützt. Die Samenbanken selbst operieren durchaus schon auf der Grundlage eugenischer Kriterien: Alter des Mannes, ethnische Herkunft, Haarfarbe, Religionszugehörigkeit, IQ. Männer können auf diese Weise 'Väter' von vielen Kindern werden; mit Eizellentransfer und Leihmutterschaft wird sich auch die Zahl der 'Mütter' vervielfältigen. 
Mittlerweile wird der Zusammenhang von Mutterschaft und Vaterschaft auf der Grundlage der zur Verfügung stehenden technischen Mittel noch weiter zergliedert und alle Elemente werden kombiniert: die Eizellen einer Frau können, extern befruchtet durch den Samen irgendeines Mannes, einer beliebigen anderen Frau eingesetzt werden, die das Kind für eine dritte Frau oder einen Mann austrägt, die oder der mit einem Mann eine Familie gründet. Es muss beachtet werden, dass dies in Deutschland so nicht möglich ist. Sowohl Eizellspende - im Gegensatz zur Samenspende - wie Leihmutterschaft sind verboten. Die gesetzlichen Krankenkassen übernehmen zudem nur bei verheirateten Paaren die Kosten der Herbeiführung einer Schwangerschaft, was somit u.a. verpartnerte Lesben und alleinstehende Frauen benachteiligt. Dies muss auch vor dem Hintergrund gesehen werden, dass es für schwule und lesbische Paare immer noch nicht möglich ist, ein Kind in Deutschland gemeinsam zu adoptieren.

Doch wie so häufig werden die technisch vorhandenen Möglichkeiten genutzt und von Marktakteuren angeboten. So führen die unterschiedlichen nationalstaatlichen Regelungen zu einer Art „Reproduktionstourismus“, die Gebärfähigkeit von Frauen kann der Ausbeutung unterworfen werden. Wie Frauen, die auf diese Weise ihre Subsistenz sichern, wird im Artikel von Veronika Siegl für Russland dargelegt. Doch es ist nicht nur die negative Seite zu betrachten: Samenbanken, künstliche Befruchtung und Leihmutterschaft ermöglichen gleichgeschlechtlichen Paaren, Kinder zu bekommen. Allerdings müssen auch hier die jeweiligen Reglungen des Landes berücksichtigt werden: In der Ukraine gibt es beispielsweise die Möglichkeit der Leihmutterschaft, sie steht allerdings schwulen und lesbischen
Paaren nicht offen. Es gibt jedoch auch Leihmutter-Agenturen, die sich speziell an bestimmte Gruppen richten, so wie Surrogate Baby in Kaliningrad an schwule Paare. Der Kostenpunkt betrug vor dem Fall des Rubels für die Paare 42.500 Euro, von denen der Leihmutter eine Aufwandsentschädigung von bis zu 20.000 Euro erhielt (Stand 2013). Die technologischen Möglichkeiten führen also zu neuartigen moralischen Konfliktkonstellationen für die gesamte Gesellschaft. Denn es sind Frauen oder gleichgeschlechtliche Paare, die sich ihren Kinderwunsch mithilfe neuer medizinischer Reproduktionstechnologien erfüllen wollen, und damit auch neue soziale Verhältnisse schaffen. Solche ausbeuterischen Verhältnisse werden von schwulen und lesbischen Paaren unterlaufen, die sich zusammentun, um gemeinsam Kinder zu bekommen und für sie zu sorgen.

Mittlerweile hat es mehrere Fälle von Uterustransplantation gegeben, sodass Frauen, die z.B. Gebärmutterhalskrebs hatten oder keinen Uterus besaßen, sich dennoch ihren Kinderwunsch erfüllen konnten. In Schweden wurden 2012 - um das Risiko der Abstoßung zu mindern - bei zwei Patientinnen die Uteri der jeweiligen Mütter verpflanzt. Es ist allerdings noch offen, ob sich diese Form der Reproduktion durchsetzen wird. Es gibt aber Andeutungen von einzelnen Medizinern, dass sie das Ziel körperexterner Uteri verfolgen, sodass irgendwann „Mütter“ und „Leihmütter“ überflüssig werden.

Neben diesen das "Leben“ allgemein betreffenden Praktiken kommt es zu einem neuen Zugriff auf das Äußere des Körpers durch die plastische Chirurgie. Der Körper selbst wird zu einem ästhetischen Projekt. Er wird nicht mehr nur durch Kleidung, Kosmetik, Tätowierung, Sport oder Fitness von außen her gestaltet, sondern unmittelbar zum Gegenstand 
der Umgestaltung. Millionen Menschen unterwerfen sich solchen Eingriffen wie Brusterweiterung oder -verkleinerung, Fettabsaugen, Facelifting, Muskelerweiterung, Verlängerung der Beine oder Umgestaltung der Nase, des Kinns oder der Füße. In vielen Fällen unterziehen sich Individuen einer ganzen Serie solcher Eingriffe und nehmen die ästhetischen Folgen wie ein künstlich wirkendes Erscheinungsbild - man denke an die vielen süffisanten Kommentare zu Nicole Kidman - oder die Risiken schlechter Heilungserfolge oder gesundheitlicher Schädigungen in Kauf. Solche in allen Altersgruppen vor allem unter Frauen verbreiteten Praktiken stellen mittlerweile ein boomendes Geschäft dar, das global betrieben wird. Diese Themen werden in den Beiträgen von Rüdiger Kunow und Frederike Offizier vertieft. Da solche Eingriffe teuer sind, entsteht auch ein regelrechter schönheitschirurgischer Tourismus. Die Zahl der entsprechenden Eingriffe nimmt in vielen Gesellschaften zu. Die Motive, sich diesen Eingriffen, die durchaus gefährlich sind, auszusetzen, sind sehr unterschiedlich. Sie können dem ästhetischen Bedürfnis nach einem besseren Aussehen oder dem Wunsch, jung zu bleiben, entsprechen; sie können, wie in dem Aufsatz von Eun-Jeung Lee ausgeführt wird, Ergebnis starken sozialen Drucks sein oder auch der Erwartung entsprechen, auf dem Arbeitsmarkt erfolgreicher zu sein.

Der biotechnologische Kapitalismus unterwirft, vermarktet und handelt mit dem Leben selbst. Die Praktiken des Humangenomprojekts, die Stammzellenforschung oder die biogenetische Behandlung von Tieren, Saatgut, Zellen oder Pflanzen sind dem Kapitalismus nicht äußerlich. Rosi Braidotti (2014: 65) spricht deswegen von der ,biogenetischen Struktur des heutigen Kapitalismus". Zu Recht weist Petra Gehring (2006) darauf hin, dass alle diese unterschiedlichen Praktiken um das „Leben" herum nicht allein durch Kommerzialisierung zu erklären sind. Immer noch machen in vielen Fällen die Unternehmen keine Gewinne. Der so unterschiedliche Zugriff auf das Leben, den Körper, die Daten und Stoffe ergibt sich aus komplexen Machtpraktiken, die im Anschluss an Michel Foucaults Arbeiten als Biomacht begriffen werden. Foucault hat den $\mathrm{Zu}$ sammenhang von Biomacht und kapitalistischer Gesellschaftsformierung immer betont: erst mit dem Kapitalismus des 19. Jahrhunderts wurde das Leben selbst in das Kalkül der Herrschaft aufgenommen, um seine Produktivität zu erhöhen und seine Entwicklung zu kontrollieren. Susanne Lettow weist in ihrem Beitrag darauf hin, dass das Verhältnis von Biokapitalismus und Biomacht immer noch zu weniggeklärt sei. Es ist auch keineswegs klar, in welcher Weise der ökonomischen Dimension Rechnung getragen werden kann. Es wird von Bioökonomie (vgl. Lettow 2012), Biowert oder Biokapital gesprochen. Sunder Rajan (2009), der von Biokapitalismus spricht, vertritt die These, dass diese neue Sphäre nicht einfach nur eine weitere Branche ist, sondern vielmehr eine Weiterentwicklung des Kapitalismus darstellt. Das Biokapital sei kein Symptom eines vollkommen neuen Stadiums des Kapitalismus, aber überdeterminiere den Kapitalismus durch „life science“, Biotech-Industrie, durch neue Eigentums- und Rechtsverhältnisse, Ausbeutungspraktiken, kulturelle Muster und Gewohnheiten (ethische Vorstellungen, Wissenschaftsverständnis), nationale oder regionale Politiken. Der Kapitalismus stellt keine einfache Einheit dar, sondern entfaltet sich durch Kapitalismen. Insofern ist der Biokapitalismus eine Fortführung und Weiterentwicklung - wie das auch für andere Bereiche gilt, die durch Informationsund Nanotechnologie oder Neuro- und 
Kognitionswissenschaften entstanden sind und ebenfalls dazu beitragen, die Gestalt des Kapitalismus zu verändern. Der Biokapitalismus, den Sunder Rajan vor Augen hat, ist der, der sich vor allem mit menschlichen Genen befasst: es wird mit Rohdaten, Algorithmen, Software-Codes gehandelt, es geht um DNA, Informationen, Proteine, Zellen oder Gewebe. Mittels biotechnischer Mittel sollen Moleküle gefunden werden, die dann zu Medikamenten weiter entwickelt werden. Im Zentrum der Bemühungen stehen Innovationen, die zu Patenten und Eigentumsrechten werden, also zu einem Monopol. Was aber hat das genau mit Kapitalismus zu tun? Wie muss man sich den Prozess der Verwertung von Kapital vorstellen? Gilt die Vorstellung von Marx noch, dass sich Kapital nur durch die Aneignung des menschlichen Arbeitsvermögens verwertet? Die Diskussion ist an diesem Punkt offensichtlich nicht sehr klar, wie Kean Birch und David Tyfield in ihrem Artikel argumentieren. Der Prozess lässt sich als eine Landnahme bezeichnen, also als eine Exploitation von Rohstoffen, die in Wert gesetzt werden. Die Bildung von Monopolen erlaubt es, mit einer Rente zu rechnen. Positionen wie die von Melinda Cooper suggerieren, dass das Leben selbst ein Mehrprodukt erzeugt, das zirkuliert werden kann. Aber daraus resultiert noch nicht die Bildung von Wert und Mehrwert im Sinn der marxschen Theorie, für die nur Arbeit wertbildend ist - und dies auch nicht als solche, sondern nur insofern, als sie ein Moment der gesellschaftlichen Gesamtarbeit ist, die privat erbracht wird und sich in Gestalt von Ware und Geld auf dem Markt in ein Verhältnis zu allen anderen privaten Arbeiten setzen muss. Birch und Tyfield ebenso wie Lettow weisen in ihren Beiträgen auf die weitgehend nur metaphorischen Anleihen bei Marx hin. Damit bleibt die Frage danach, was am
Biokapitalismus das spezifisch Kapitalistische ist, immer noch offen. Wo wird also der Wert und Mehrwert erzeugt? Birch und Tyfield antworten mit dem Hinweis auf die Wissensarbeit. Hier findet die Arbeit der Aneignung und Transformation von Natur statt. Aber das würde bedeuten, dass die Menschen, von denen die DNA, die Gewebe, die Organe stammen, nur als passive Rohstoffe betrachtet werden. Doch auch die Erzeugung und Erhaltung der Körper selbst ist Arbeit. FeministInnen haben dies mit Blick auf die Reproduktionsarbeit seit Langem gesagt. Die Geburt und die vielen Care-Aktivitäten, die vor allem von Frauen geleistet werden, wurden, so die Kritik an der marxschen Theorie, außer Betracht gelassen. Jetzt rückt die Produktion und Reproduktion des biologischen Körpers auf eine weitere und neue Weise in den Blick. Denn indem sich ein Markt herausbildet und der Körper und seine Form, seine Organe, Gewebe, DNA-Sequenzen selbst der Warenform unterworfen werden, kann davon gesprochen werden, dass es sich um Arbeit handelt, die alle diese "Waren“ erzeugt. Das menschliche Arbeitsvermögen, das hier der Warenform unterworfen wird, ist die Fähigkeit zur Erzeugung des Lebens selbst: also die Arbeit der Erhaltung, des Wachstums des Körpers, der bestimmte Produkte für die biotechnische Nutzung erzeugt. Auch hier kommt es am Ende zu dem Widerspruch, auf den Marx mit Blick auf die Aneignung der äußeren Natur hingewiesen hat: Die Natur wird degradiert und zerstört. Denn die Individuen erzeugen sich, um vitale Funktionen zu verkaufen: Gebärfähigkeit, Organe, Informationen, Stoffe. Was darüber hinaus am Markt nicht verwendbar ist, was keinen Gebrauchswert für andere Marktteilnehmer hat, ist unnütz. Was geschieht mit dem Rest, der nach einer Organentnahme, nach einer Leihmutterschaft, nach 
entsprechender Hormonbehandlung und chirurgischen Eingriffen, nach schönheitschirurgischen Eingriffen übrig bleibt? Die Körper werden zu Wegwerfkörpern, zu Abfall. Mit der Rückwendung des Verwertungsprozesses auf die innere Natur wird der Produktionsprozess für viele Menschen unmittelbar gefährlich. Das alles könnten Übergänge darstellen. Zum einen in die Richtung einer effektiveren Vernutzung menschlicher Körper; zum anderen könnten mit weiteren medizinischen und gentechnischen Fortschritten Eingriffe wie die Organtransplantation überflüssig werden.

Die Tendenz zur Inwertsetzung der Körper und des Lebens stellt eine Herausforderung dar und wirft viele Fragen auf. Dazu gehört auch die nach dem Maßstab der Kritik. Es ist nicht zu leugnen, dass Menschen sich nach einer Schönheitsoperation besser, schöner, sicherer fühlen können oder sich ihre Arbeitsmarktchancen verbessern; dass sie sich den Wunsch nach einem eigenen Kind erfüllen können; dass Transgenderpersonen sich nach chirurgischen Eingriffen mit sich selbst identischer fühlen; dass Organtransplantationen Leben retten. Die Bioethik ist selbst ein Geschäftszweig, der von Unternehmen genutzt wird, um Akzeptanz zu erzeugen. Aber die kritische Gesellschaftstheorie muss auf diese Fragen antworten und solche positiven Aspekte in die Gesellschaftskritik miteinbeziehen. Der Rückgriff auf ein humanistisches, naturalistisches Bild vom Menschen, also einem Menschen, der von der Natur getrennt wird, von dem angenommen wird, dass er ein sich selbst bewusstes, autonomes Subjekt sei, das durch diese neuen Entwicklungen nur beeinträchtigt wird, reicht nicht aus. Diese Auffassung wird nicht nur durch die Lebenswissenschaften infrage gestellt. In Anschluss an Autor_innen wie Nikolas Rose lässt sich die Frage stellen, welche emanzipatorischen Potenziale die neuen Biotechnologien, überhaupt erst hervorbringen. Donna Haraway hatte schon in den 1980er Jahren davon gesprochen, dass Individuen aufgrund der vielen Prothesen und Eingriffe schon längst Cyborgs sind. Die Entwicklungen verlangen also auch neue Formen der kritischen Auseinandersetzung auf dem Niveau der durch die neuen Technologien ermöglichten gesellschaftlichen Praktiken. Rosi Braidotti (2014) hat diese Konstellation als posthumanistisch gekennzeichnet. Gerade die Tatsache, dass die Menschen sich das Leben unterwerfen, könnte auch die radikale Möglichkeit dafür schaffen, die Spezies Mensch selbst als Natur zu erkennen. Das Leben der Menschen könnte sich mit dem Schicksal anderer Arten verbinden und sich in ein neues Verhältnis zur inneren und äußeren Natur setzen.

Neben dem Schwerpunkt zu Biokapitalismus diskutieren wir in diesem Heft drei weitere Themen. Um das rassismustheoretische Konzept „Critical Whiteness“ entzündeten sich in den vergangenen Jahren kontroverse und heftige Diskussionen. Im Mittelpunkt stand dabei jedoch vor allem die politische Praxis bestimmter Akteure, die sich auf das Konzept berufen, weniger die theoretische und politische Anlage des Konzepts selbst. In seinem Beitrag zu diesem Heft setzt sich Serhat Karakayalı mit den Schlüsselbegriffen des Konzepts wie Normen, Privilegien und „awareness“ auseinander und diskutiert deren epistemologische und identitätstheoretische Konsequenzen. Die theoretischen und politischen „Engführungen“ der CriticalWhiteness-Strömung deutet er als Symptom und Erbschaft der gescheiterten Fusion von Marxismus und Rassismusanalyse.

Als „Einsprüche“ veröffentlichen wir immer wieder Texte, in denen Stellungnahmen zu aktuellen Ereignissen oder 
Debatten formuliert werden, und dies häufig auch in polemischer Weise. Diesmal schreibt Alp Kayserilioğlu zu dem Pariser Massaker bei Charlie Hebdo und in einem koscheren Supermarkt. Anders als in vielen aktuellen Stellungnahmen beschränkt er sich nicht darauf, den Wert der Meinungsfreiheit hochzuhalten, sondern stellt Fragen nach politischen Hintergründen und absehbaren Folgen. Es geht also sowohl um die Politik westlicher Länder nach dem Zusammenbruch der UdSSR, insbesondere der USA und ihrer direkten oder indirekten Förderung des „Politischen Islam", als auch um die soziale Ausgrenzung migrantischer Jugendlicher in westlichen Metropolen, die einigen von ihnen islamistische Gruppierungen attraktiv erscheinen lässt. $\mathrm{Ob}$ „der Imperialismus“ als Erklärung dafür taugt, wurde in der Redaktion äußerst kontrovers diskutiert. Wir möchten mit der Veröffentlichung dieses Textes, in dem viele uns zentral erscheinende Themen angeschnitten werden, eine Diskussion in Gang setzen, in der weiter nach Antworten auf diese brennenden Fragen gesucht wird.

Angesichts der Auseinandersetzungen in und um die Ukraine geht Klaus
Müller der Frage nach, warum das ,state building" in der Ukraine gescheitert ist und spannt den Bogen von der CliquenHerrschaft der Oligarchen, die sich nach 1990 entwickelte, zu den geopolitischen Konfliktachsen, die die innenpolitischen Konfliktlagen der Ukraine überlagern. Müller weist historisch nach, dass es vor allem die spät gespielte NationalismusKarte war, die Ethnisierung des Konflikts war, die zur Eskalation beitrug - provoziert durch die Interventionen der EU und der USA.

Alex Demirović und Mariana Schütt (Für die Redaktion)

\section{Literatur}

Braidotti, Rosi (2014): Posthumanismus. Frankfurt/M-New York.

Gehring, Petra (2006): Was ist Biomacht? Vom zweifelhaften Mehrwert des Lebens. Frankfurt/M-New York.

Lettow, Susanne (Hrsg.)(2012): Bioökonomie. Die Lebenswissenschaften und die Bewirtschaftung der Körper. Bielefeld.

Sunder Rajan, Kaushik (2009): Biokapitalismus. Werte im postgenomischen Zeitalter. Frankfurt/M. 